\title{
Factors associated with treatment outcomes of patients hospitalized with severe maxillofacial infections at a tertiary center
}

\author{
Hye-Won Kim, Chul-Hwan Kim \\ Department of Oral and Maxillofacial Surgery, College of Dentistry, Dankook University, Cheonan, Korea
}

\begin{abstract}
J Korean Assoc Oral Maxillofac Surg 2021;47:197-208)
Objectives: The purpose of this retrospective study was to evaluate the variables associated with length of stay (LOS), hospital costs, intensive care unit (ICU) use, and treatment outcomes in patients hospitalized for maxillofacial infections at a tertiary medical center in South Korea.

Materials and Methods: A retrospective chart review was conducted for patients admitted for treatment of maxillofacial infections at Dankook University Hospital from January 1, 2011 through September 30, 2020. A total of 390 patient charts were reviewed and included in the final statistical analyses.

Results: Average LOS and hospital bill per patient of this study was 11.47 days, and $\$ 4,710,017.25$ (\$4,216.67), respectively. Of the 390 subjects, $97.3 \%$ were discharged routinely following complete recovery, $1.0 \%$ expired following treatment, and $0.8 \%$ were transferred to another hospital. In multivariate linear regression analyses to determine variables associated with LOS, admission year, infection side, Flynn score, deep neck infection, cardiovascular disease, admission C-reactive protein (CRP) and glucose levels, number and length of surgical interventions, tracheostomy, time elapsed from admission to first surgery, and length of ICU stay accounted for $85.8 \%$ of the variation. With regard to the total hospital bill, significantly associated variables were age, type of insurance, Flynn score, number of comorbidities, admission CRP, white blood cell, and glucose levels, admission temperature, peak temperature, surgical intervention, the length, type, and location of surgery, tracheostomy, time elapsed from admission to first surgery, and length of ICU use, which accounted for $90.4 \%$ of the variation. Age and ICU use were the only variables significantly associated with unfavorable discharge outcomes in multivariate logistic regression analysis.

Conclusion: For successful and cost-effective management of maxillofacial infections, clinicians to be vigilant about the decision to admit patients with maxillofacial infections, perform appropriate surgery at an adequate time, and admit them to the ICU.
\end{abstract}

Key words: Abscess, Bacterial infections, Retropharyngeal abscess, Maxillofacial surgery, Antibiotics

[paper submitted 2021. 3. 3 / revised 2021. 5. 6 / accepted 2021. 5. 11]

\section{Introduction}

Maxillofacial abscess or cellulitis is an infection of the soft tissues of the face and neck area that arises from odontogenic or nonodontogenic causes ${ }^{1}$. During the initial stage, maxillofacial infections are usually mild and prevention and treatment are often straightforward and relatively inexpensive, consisting of appropriate surgical procedures and antibiotics

\section{Chul-Hwan Kim \\ Department of Oral and Maxillofacial Surgery, College of Dentistry, Dankook University, 119 Dandae-ro, Dongnam-gu, Cheonan 31116, Korea TEL: +82-41-550-1996 \\ E-mail:kimchoms@dankook.ac.kr \\ ORCID: https://orcid.org/0000-0002-5199-2420}

(c) This is an open-access article distributed under the terms of the Creative Commons Attribution Non-Commercial License (http://creativecommons.org/ licenses/by-nc/4.0/), which permits unrestricted non-commercial use, distribution, and reproduction in any medium, provided the original work is properly cited. Copyright (C) 2021 The Korean Association of Oral and Maxillofacial Surgeons. All rights reserved. in an outpatient clinic setting. However, infections that spread beyond the initial border can lead to life-threatening maxillofacial complications such as necrotizing fasciitis, cavernous sinus thrombosis, airway obstruction, mediastinitis, and sepsis, and therefore require hospital admission ${ }^{1,2}$. This can result in delayed recovery, prolonged hospital stay, intensive care unit (ICU) admission, increased risk of mortality, and a substantial increase in the overall costs of treatment ${ }^{2,3}$. Severe maxillofacial infections can therefore be a significant economic burden to both health care facilities and patients.

However, there is a paucity of literature examining the financial aspects of hospitalization of patients with head and neck infections, and the parameters of these important cost variables are poorly understood. The few studies regarding hospital costs that have been performed have been limited to Western populations, but such results are likely biased due to regional differences in the epidemiology of maxillofacial 
infections and health insurance systems ${ }^{1-10}$. In this era in which cutting budgets is considered important, it is crucial that surgeons and hospitals strive to minimize costs while still maintaining the highest level of patient care. Objective data regarding variables associated with increased hospitalization costs are therefore required. An epidemiologic study to identify the distinct characteristics of severe maxillofacial infections in Asian countries would help improve overall outcomes and reduce overall health care costs.

The objective of this retrospective study was to describe the demographic characteristics, treatment outcomes, and hospital costs of patients hospitalized in a tertiary center in South Korea for the treatment of severe maxillofacial infections, and to identify the associations between these treatment outcomes and diverse social, patient health, and treatmentrelated variables.

\section{Materials and Methods}

\section{Study design}

After obtaining approval from the Institutional Review Board of Dankook Dental University Hospital (No. DKUDH IRB 2020-09-007), records of patients admitted to the Oral and Maxillofacial Surgery (OMFS) Department, Dankook University Hospital, for the treatment of maxillofacial infections from January 2011 through September 2020 were reviewed. The billing department performed a computer search of billing data attached to the electronic medical record numbers of patients with the relevant diagnostic codes from the seventh revision of the Korea Informative Classification of Diseases (KCD7). KCD7 codes used to identify relevant patients were K04.6 (periapical abscess with sinus), K04.7 (periapical abscess without sinus), K05.21 (periodontal abscess of gingival origin with sinus), K11.3 (abscess of the salivary gland), K12.2 (cellulitis and abscess of the mouth), K14.0 (abscess and ulceration of the tongue), J36 (peritonsillar abscess), J39.0 (retropharyngeal and parapharyngeal abscess), and J39.1 (other abscess of the pharynx). Retrieved positive records were then cross-checked with the relevant codes to ensure that admissions were related to a maxillofacial infection. An additional, complete manual review of patient admission data was also performed to identify additional records not identified by the electronic search process. Exclusion criteria for this study were patients treated only in an outpatient setting, incomplete data acquisition from electronic charts, patients not yet discharged from the hos- pital, patients who were initially admitted for reasons other than maxillofacial infections but who developed infection secondary to other maxillofacial surgeries, and patients for whom the OMFS service was not consulted. To focus on the characteristics and outcomes of more severe, potentially fatal infections, the current study was limited to cases that required hospital admission for treatment. To ensure that the exclusion criteria were applied and that recording of the relevant data was accurate, each chart was reviewed twice by two separate reviewers. At the reviewing stage, the reviewers were blinded to the hospital bills of each subject to eliminate the possibility of bias in collecting data. Names of the subjects were not revealed to protect personal information.

\section{Independent variables}

All variables extracted from patient records were recorded and organized on an electronic Microsoft Excel 2021 (Microsoft, Redmond, WA, USA) spreadsheet. Independent variables examined in the present study comprised a heterogeneous set of demographic, individual health status, hospital course, infection, and treatment-related variables.

Demographic variables were age, sex, ethnicity (Korean or not Korean), insurance coverage status (National Health Insurance [NHI], medical aid, or uninsured), and admission year. Age, sex, and ethnicity were determined based upon information in patients' medical records. Information regarding insurance status was provided by the billing department. Admission year was determined based on admission and discharge dates. When the patient was admitted at the end of one year and discharged the next year, the admission year was considered the year with the longer length of stay (LOS).

Variables related to an individual's health status were the presence of any kind of comorbidity, the number of comorbidities, smoking, and the burden of each type of comorbid condition. All these variables were noted as written in the nursing admission assessment form. To assess the burden of comorbid conditions such as diabetes, endocrine disease, nephrotic disease, hepatic disease, cardiovascular disease, and psychological disease, each condition was tested bivariately and only those associated significantly with outcomes were included in the final statistical analysis.

Hospital course-related variables were route of admission (via outpatient clinic or emergency room [ER]), hospital arrival time (morning [00:00 to $08: 00]$, day [08:00 to $16: 00$ ], or evening [16:00 to 24:00]), and time elapsed from symptom onset to admission, from admission to first surgery, and from 
symptom onset to first surgery (in days). Route of admission and hospital arrival time were determined from information provided by the billing department. Date of symptom onset was recorded as described by the patients at the time of the first ER or outpatient clinic visit. Date of the first surgery was determined from the procedure and surgery notes.

Infection-related variables included the infection's etiology (odontogenic or non-odontogenic), number of infected anatomical spaces, infected location, presence of deep neck space infection, presence of necrotizing fasciitis, Flynn score, C-reactive protein (CRP) level, white blood cell (WBC) count, glucose level upon admission, body temperature upon admission, and peak body temperature during hospital stay. Information regarding the infection's etiology and spaces involved was determined by concomitant review of discharge summaries and enhanced neck computed tomography scans. An odontogenic infection severity score was assigned to each infection based on a scoring system suggested in a previous study by Flynn et al. ${ }^{4}$, in which each infected fascial space is scored on the basis of its proximity to the airway, other vital structures, and likelihood of preventing airway access; the total score is the aggregate of these values. Thus the vestibular, subperiosteal, infraorbital, canine, and buccal spaces were assigned a score of 1, the submandibular, submental, sublingual, and masticatory spaces a score of 2 , and the lateral pharyngeal, retropharyngeal, pretracheal, mediastinal, and intracranial spaces a score of 3 . Body temperatures were determined through review of the patient's daily clinical examination form, and were categorized as $37.5^{\circ} \mathrm{C}$ or more or less than $37.5^{\circ} \mathrm{C}$.

Treatment-related variables included antibiotic regimen, performance of incision and drainage, tracheostomy, any type of surgical intervention after admission, general anesthesia (GA), additional surgery, additional operating room (OR) uses, total number of OR visits, surgeries, type and location of surgeries received, ICU use, and length of ICU stay. Antibiotic regimen was categorized based upon the most commonly utilized combinations of antibiotics during the reviewed period, and was clarified by reviewing the daily prescription data. All information regarding surgical interventions and GA was obtained by comprehensive review of the information recorded on emergency department records, daily procedure notes, daily inpatient notes, surgery notes, anesthesia notes, and discharge summaries. Among surgeryrelated variables, length of surgery was calculated as the difference between the recorded 'procedure start' and 'procedure end' times on the anesthesia note. Information regarding ICU stay was provided by the billing department.

\section{Outcome variables}

Primary outcome variables of interest were total hospital charges, LOS in hospital (in days), mean hospital bill per one hospital admission day, use of ICU services, and disposition status (routine discharge following complete recovery, transfer to another hospital, discharge against medical advice, death). Total hospital charges were determined as the sum of the final amount billed to the patient and the bill covered by the NHI service in Korean Won, which was obtained from data provided by the billing department. LOS indicates the total number of days that the patient stayed in the hospital since admission. Mean hospital bill per one admission day was calculated as the total hospital bill divided by the total LOS in days. Disposition status was copied from the discharge records.

\section{Statistical analysis}

All retrieved data were compiled, matched with the outcome data, and imported into IBM SPSS Statistics (ver. 27.0; IBM, Armonk, NY, USA) for statistical analyses. Descriptive statistics were computed for all variables. Because data regarding hospital charges were highly skewed and not normally distributed, the billing data was log-transformed to adjust for violation of the normality assumption. For preliminary univariable analysis, data were analyzed using the $t$-test, Mann-Whitney test, analysis of variance, Kruskal-Wallis test, Pearson's correlation analysis, $\chi^{2}$ test, and Fisher's exact test as appropriate for the bivariate analysis performed. A $P$-value less than 0.05 was considered to be statistically significant. All variables with statistically significant associations with the dependent variables $(P<0.05)$ from the initial univariable analysis were used to formulate a preliminary multiple linear regression model for the continuous dependent variables, and a preliminary multiple logistic regression model for the binary dependent variables. The final multiple regression models included all variables from the preliminary model with $P$-values lower than 0.05 . In all regression models, adjustments were made for the clustering of outcomes. All statistical analyses were two-sided. 


\section{Results}

\section{Patient data}

A total of 401 patients were identified via the initial electronic chart review. Of these patients, 390 (205 male, 185 female) met the inclusion criteria and were thus included in the final analysis. The remaining 11 patients were excluded from the study because only incomplete data regarding the type or location of surgery could be acquired from the electronic charts $(n=6)$, the patients were initially admitted for reasons other than maxillofacial infection $(n=4)$, or were not yet discharged $(\mathrm{n}=1)$.

\section{Demographics and insurance status}

Demographic traits of the reviewed subjects are summarized in Table 1. In total, $52.6 \%$ of subjects were male and $47.4 \%$ were female. Mean age at presentation was $54.69 \pm 20.83$ years (range, $4-90$ years). Only $2.1 \%$ of subjects were not Korean. Of the patients, $93.1 \%$ were insured by the NHI, $5.9 \%$ by medical aid, and the remaining $1.0 \%$ were not insured. The number of patients admitted each year did not differ significantly.

\section{Health status}

Data related to the health status of the patients included in this study are summarized in Table 1. Among the population studied, $59.0 \%$ of patients had multiple comorbidities. Mean number of comorbid conditions present per patient was $1.21 \pm 1.33$ (range, $0-6$ ). One hundred thirty-nine patients $(35.6 \%)$ were cigarette smokers.

\section{Hospital course}

Descriptive statistics pertaining specifically to subjects' hospital course are depicted in Table 1. Most patients were admitted via the ER (83.3\%), and $67.9 \%$ of the initial visits were made during the daytime. Mean number of days that elapsed from symptomatic onset to admission was 7.23 \pm 7.97 days (range, 0-60 days), to first surgery was $7.89 \pm 7.55$ days (range, 0-50 days), and mean number of days from admission to first surgery was $0.78 \pm 1.79$ days (range, $0-12$ days).

\section{Etiology, location, and severity of infection}

Relevant information regarding the characteristics of the infections are illustrated in Table 1. Average number of infected anatomical spaces per patient was $2.16 \pm 1.47$ (range, 1-9), mean Flynn score was $4.13 \pm 3.13$ (range, 1-19), and there were infections in both sides of the face and neck in 26 cases $(6.7 \%)$. Deep neck involvement was noted in 51 patients $(13.1 \%)$, while the presence of necrotizing fasciitis was confirmed in 13 patients (3.3\%). Only 11 cases $(2.8 \%)$ were due to non-odontogenic causes. Mean CRP, WBC, and serum glucose levels upon admission were $13.12 \mathrm{mg} / \mathrm{dL}, 13.91 \times 10^{3} /$ $\mu \mathrm{L}, 140.25 \mathrm{mg} / \mathrm{dL}$, respectively. While only 64 cases $(16.4 \%)$ presented to the hospital with a body temperature higher than $37.5^{\circ} \mathrm{C}, 149$ patients (38.2\%) developed fever during the hospital stay.

\section{Treatment}

Descriptive data regarding treatment-related variables are shown in Table 1. Most patients underwent incision and drainage (82.3\%) and surgery after admission (66.9\%) in the OR (40.0\%), but did not require operation under GA (62.1\%), additional surgery $(81.8 \%)$, additional GA $(95.6 \%)$, tracheostomy $(96.7 \%)$, or any other surgical interventions other than incision and drainage (67.2\%). Of the 390 patients, 62 patients $(15.9 \%)$ did not receive any kind of surgical treatment at all but were treated conservatively with intravenous antibiotics. The most common antibiotic regimen used to treat the majority of the reviewed infections (73.8\%) was concomitant use of amoxicillin-sulbactam or ceftriaxone with clindamycin. Only 46 cases $(11.8 \%)$ required a stay in the ICU for more than one day, and the average LOS in the ICU was $1.12 \pm 6.09$ days (range, 0-94 days).

\section{Total hospital bill, LOS, and treatment outcomes}

Mean hospital bill was $\$ 4,710,017.25$ (4,216.67 USD),

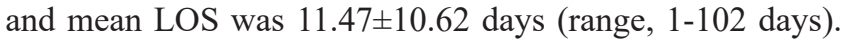
Mean hospital bill incurred per one admission day was \#386,225.64 (345.77 USD). Approximately $96.9 \%$ of all hospitalizations were discharged routinely following treatment; of those that were not routinely discharged, $1.3 \%$ were discharged against medical advice, $0.8 \%$ were transferred to another hospital, and $1.0 \%$ died of the disease. 
Table 1. Descriptive statistics of the study population $(n=390)$

\begin{tabular}{|c|c|}
\hline Variable & Value \\
\hline \multicolumn{2}{|l|}{ Sex } \\
\hline Male & $205(52.6)$ \\
\hline Female & $185(47.4)$ \\
\hline Age $(y r)$ & $54.69 \pm 20.83$ \\
\hline \multicolumn{2}{|l|}{ Ethnicity } \\
\hline Korean & $382(97.9)$ \\
\hline Not Korean & $8(2.1)$ \\
\hline \multicolumn{2}{|l|}{ Insurance } \\
\hline NHI & $363(93.1)$ \\
\hline Medial aid & $23(5.9)$ \\
\hline Not insured & $4(1.0)$ \\
\hline \multicolumn{2}{|l|}{ Admission year } \\
\hline 2011-2012 & $69(17.7)$ \\
\hline 2013-2014 & $65(16.7)$ \\
\hline $2015-2016$ & $88(22.6)$ \\
\hline $2017-2018$ & $81(20.8)$ \\
\hline $2019-2020$ & $87(22.3)$ \\
\hline \multicolumn{2}{|l|}{ Medically compromised } \\
\hline Present & $230(59.0)$ \\
\hline Not present & $160(41.0)$ \\
\hline No. of comorbidities & $1.21 \pm 1.33$ \\
\hline \multicolumn{2}{|l|}{ Smoking } \\
\hline Yes & $251(64.4)$ \\
\hline No & $139(35.6)$ \\
\hline \multicolumn{2}{|l|}{ Route of admission } \\
\hline ER & $325(83.3)$ \\
\hline Outpatient clinic & $65(16.7)$ \\
\hline \multicolumn{2}{|l|}{ Hospital arrival time } \\
\hline Day & $265(67.9)$ \\
\hline Evening & $89(22.8)$ \\
\hline Night & $36(9.2)$ \\
\hline $\begin{array}{l}\text { Time from symptomatic onset to } \\
\text { hospitalization (day) }\end{array}$ & $7.23 \pm 7.97$ \\
\hline $\begin{array}{l}\text { Time from onset of symptoms to first } \\
\text { surgery (day) }\end{array}$ & $7.89 \pm 7.55$ \\
\hline Time from admission to first surgery (day) & $0.78 \pm 1.79$ \\
\hline \multicolumn{2}{|l|}{ Odontogenic etiology } \\
\hline Yes & $379(97.2)$ \\
\hline No & $11(2.8)$ \\
\hline No. of infected spaces & $2.16 \pm 1.47$ \\
\hline \multicolumn{2}{|l|}{ Location of infection } \\
\hline Single & $355(91.0)$ \\
\hline Middle & $9(2.3)$ \\
\hline Both & $26(6.7)$ \\
\hline \multicolumn{2}{|l|}{ Deep neck infection } \\
\hline Present & $339(86.9)$ \\
\hline Not present & $51(13.1)$ \\
\hline \multicolumn{2}{|l|}{ Fasciitis } \\
\hline Present & $377(96.7)$ \\
\hline Not present & $13(3.3)$ \\
\hline Flynn score & $4.13 \pm 3.13$ \\
\hline Admission C-reactive protein level (mg/dL) & $13.12 \pm 10.25$ \\
\hline $\begin{array}{l}\text { Admission white blood cell count } \\
(\times 1,000 \text { cells } / \mu \mathrm{L})\end{array}$ & $13.91 \pm 5.40$ \\
\hline Admission blood glucose level (mg/dL) & $140.25 \pm 70.05$ \\
\hline \multicolumn{2}{|l|}{ Admission temperature } \\
\hline$<37.5^{\circ} \mathrm{C}$ & $326(83.6)$ \\
\hline$>37.5^{\circ} \mathrm{C}$ & $64(16.4)$ \\
\hline \multicolumn{2}{|l|}{ Peak temperature during stay } \\
\hline$<37.5^{\circ} \mathrm{C}$ & $241(61.8)$ \\
\hline$>37.5^{\circ} \mathrm{C}$ & $149(38.2)$ \\
\hline \multicolumn{2}{|l|}{ Antibiotic regimen } \\
\hline $\begin{array}{l}\text { Single (amoxicillin-sulbactam or } \\
\text { ceftriaxone sodium hydrate) }\end{array}$ & $35(9.0)$ \\
\hline $\begin{array}{l}\text { Double (amoxicillin-sulbactam/ } \\
\text { ceftriaxone sodium hydrate+clindamycin) }\end{array}$ & $288(73.8)$ \\
\hline $\begin{array}{l}\text { Triple } 1 \text { (amoxicillin-sulbactam/ } \\
\text { ceftriaxone+clindamycin+metronidazole) }\end{array}$ & $36(9.2)$ \\
\hline $\begin{array}{l}\text { Triple } 2 \text { (amoxicillin-sulbactam/ } \\
\text { ceftriaxone+clindamycin with quinolones/ } \\
\text { amikacin) }\end{array}$ & $21(5.4)$ \\
\hline $\begin{array}{l}\text { Use of vancomycin or meropenem } \\
\text { with concomitant use of other antibiotics }\end{array}$ & $10(2.6)$ \\
\hline
\end{tabular}

Table 1. Continued

\begin{tabular}{|c|c|}
\hline Variable & Value \\
\hline \multicolumn{2}{|l|}{ I\&D } \\
\hline Performed & $321(82.3)$ \\
\hline Not performed & $69(17.7)$ \\
\hline \multicolumn{2}{|l|}{ Tracheostomy } \\
\hline Performed & $13(3.3)$ \\
\hline Not performed & $377(96.7)$ \\
\hline \multicolumn{2}{|l|}{ Surgery after admission } \\
\hline Performed & $261(66.9)$ \\
\hline Not performed & $129(33.1)$ \\
\hline \multicolumn{2}{|l|}{ General anesthesia } \\
\hline Performed & $148(37.9)$ \\
\hline Not performed & $242(62.1)$ \\
\hline \multicolumn{2}{|l|}{ Additional surgery } \\
\hline Performed & $71(18.2)$ \\
\hline Not performed & $319(81.8)$ \\
\hline \multicolumn{2}{|l|}{ Additional trip to $\mathrm{OR}$} \\
\hline Yes & $17(4.4)$ \\
\hline No & $373(95.6)$ \\
\hline No. of OR uses & $0.43 \pm 0.60$ \\
\hline Length of GA & $20.80 \pm 39.95$ \\
\hline No. of operations & $1.05 \pm 0.66$ \\
\hline \multicolumn{2}{|l|}{ Operation type } \\
\hline None & $62(15.9)$ \\
\hline I\&D alone & $262(67.2)$ \\
\hline I\&D \& extraction (without other surgery) & $30(7.7)$ \\
\hline $\begin{array}{l}\text { I\&D \& surgery (other than extraction or } \\
\text { tracheostomy) }\end{array}$ & $20(5.1)$ \\
\hline $\begin{array}{l}\text { I\&D \& tracheostomy (with or without other } \\
\text { surgeries) }\end{array}$ & $9(2.3)$ \\
\hline Other surgeries (with or without extraction) & $7(1.8)$ \\
\hline \multicolumn{2}{|l|}{ Location of surgery } \\
\hline Outpatient clinic before admission & $67(17.2)$ \\
\hline ER & $37(9.5)$ \\
\hline Outpatient clinic after admission & $68(17.4)$ \\
\hline OR & $156(40.0)$ \\
\hline No surgery & $62(15.9)$ \\
\hline \multicolumn{2}{|l|}{ ICU use } \\
\hline Yes & $46(11.8)$ \\
\hline No & $344(88.2)$ \\
\hline Length of ICU stay (day) & $1.12 \pm 6.09$ \\
\hline Length of hospital stay (day) & $11.47 \pm 10.62$ \\
\hline \multirow[t]{3}{*}{ Total bill (Korean Won) } & $4,710,017.25 \pm$ \\
\hline & $386,225.64$ \\
\hline & $(\$ 4,216.67)$ \\
\hline \multirow{3}{*}{$\begin{array}{l}\text { Average bill per one hospital day } \\
\text { (Korean Won) }\end{array}$} & $386,225.64 \pm$ \\
\hline & $188,833.73$ \\
\hline & $(\$ 345.77)$ \\
\hline \multicolumn{2}{|l|}{ Treatment outcome } \\
\hline Total recovery & $378(96.9)$ \\
\hline Discharge against medical advice & $5(1.3)$ \\
\hline Transfer to another hospital & $3(0.8)$ \\
\hline Death & $4(1.0)$ \\
\hline
\end{tabular}

(NHI: National Health Insurance, ER: emergency room, I\&D: incision and drainage, OR: operation room, GA: general anesthesia, ICU: intensive care unit)

Values are presented as number $(\%)$ or mean \pm standard deviation.

Hye-Won Kim et al: Factors associated with treatment outcomes of patients hospitalized with severe maxillofacial infections at a tertiary center. J Korean Assoc Oral Maxillofac Surg 2021

\section{Univariable and multivariable analyses}

Univariate analysis results regarding the associations between the independent variables and LOS, total hospital bill, average hospital bill per day, ICU stay, and discharge outcomes other than routine discharge are summarized in Table 2. 
Table 2. $P$-values from univariable analyses of selected variables $(n=390)$

\begin{tabular}{|c|c|c|c|c|c|}
\hline Independent variable & Length of stay & $\begin{array}{l}\text { Total } \\
\text { hospital bill }\end{array}$ & $\begin{array}{l}\text { Average bill } \\
\text { per day }\left(\log _{2}\right)\end{array}$ & ICU stay & $\begin{array}{l}\text { Other outcomes vs } \\
\text { complete recovery }\end{array}$ \\
\hline Sex (binary) & $0.01^{1, *}$ & $0.132^{1}$ & $0.125^{1}$ & $0.189^{2}$ & $0.857^{2}$ \\
\hline Age (continuous) & $<0.001^{3, *}$ & $<0.001^{3, *}$ & $0.024^{3, *}$ & $<0.001^{1, *}$ & $0.001^{1, *}$ \\
\hline Ethnicity (binary) & $0.47^{4}$ & $0.718^{4}$ & $0.065^{4}$ & $0.241^{2}$ & $>0.999^{2}$ \\
\hline Insurance (categorical) & $0.74^{5}$ & $0.024^{5, *}$ & $0.006^{5, *}$ & $0.028^{6, *}$ & $0.118^{6}$ \\
\hline Admission year (categorical) & $0.02^{7, *}$ & $<0.001^{7, *}$ & $<0.001^{7, *}$ & $0.323^{6}$ & $0.080^{6}$ \\
\hline Medically compromised (binary) & $<0.001^{1, *}$ & $<0.001^{1, *}$ & $0.023^{1, *}$ & $0.002^{2, *}$ & $0.133^{2}$ \\
\hline No. of comorbidities (continuous) & $<0.001^{3, *}$ & $<0.001^{3, *}$ & $<0.001^{3, *}$ & $<0.001^{1, *}$ & $0.04^{1, *}$ \\
\hline Smoking (binary) & $0.967^{1}$ & $0.767^{1}$ & $0.633^{1}$ & $0.599^{2}$ & $0.761^{2}$ \\
\hline DM (binary) & $0.022^{1, *}$ & $0.009^{1, *}$ & $0.655^{1}$ & $0.374^{2}$ & $>0.999^{2}$ \\
\hline Endocrine disease (binary) & $0.004^{1, *}$ & $0.001^{1, *}$ & $0.221^{1}$ & $0.109^{2}$ & $0.202^{2}$ \\
\hline Cardiovascular disease (binary) & $<0.001^{1, *}$ & $<0.001^{1, *}$ & $0.065^{1}$ & $0.031^{2, *}$ & $0.018^{2, *}$ \\
\hline Neural disease (binary) & $0.098^{4}$ & $0.028^{4, *}$ & $0.169^{4}$ & $0.041^{2, *}$ & $0.028^{2, *}$ \\
\hline Route of admission (binary) & $0.004^{1, *}$ & $<0.001^{1, *}$ & $<0.001^{1, *}$ & $0.005^{2, *}$ & $0.23^{2}$ \\
\hline Hospital arrival time (categorical) & $0.432^{7}$ & $0.072^{7}$ & $0.103^{7}$ & $0.190^{6}$ & $0.155^{6}$ \\
\hline $\begin{array}{l}\text { Time from symptomatic onset to } \\
\text { hospitalization (continuous) }\end{array}$ & $0.518^{3}$ & $0.179^{3}$ & $0.582^{3}$ & $0.424^{1}$ & $0.707^{1}$ \\
\hline $\begin{array}{l}\text { Time from onset of symptoms to first surgery } \\
\text { (continuous) }\end{array}$ & $0.625^{3}$ & $0.736^{3}$ & $0.405^{3}$ & $0.444^{1}$ & $0.348^{1}$ \\
\hline $\begin{array}{l}\text { Time from admission to first surgery } \\
\text { (continuous) }\end{array}$ & $<0.001^{3, *}$ & $<0.001^{3, *}$ & $0.179^{3}$ & $0.656^{1}$ & $0.346^{1}$ \\
\hline Etiology (binary) & $0.02^{4, *}$ & $0.003^{4, *}$ & $0.177^{4}$ & $>0.999^{2}$ & $>0.999^{2}$ \\
\hline No. of infected spaces (continuous) & $<0.001^{3, *}$ & $<0.001^{3, *}$ & $<0.001^{3, *}$ & $<0.001^{1, *}$ & $0.107^{1}$ \\
\hline Location of infection (categorical) & $0.003^{7}, *$ & $<0.001^{7, *}$ & $0.059^{7}$ & $<0.001^{6 * *}$ & $0.612^{6}$ \\
\hline Deep neck infection (binary) & $0.005^{1, *}$ & $<0.001^{1, *}$ & $<0.001^{1, *}$ & $<0.001^{2} * *$ & $0.198^{2}$ \\
\hline Fasciitis (binary) & $0.013^{1, *}$ & $<0.001^{1, *}$ & $<0.001^{1, *}$ & $<0.001^{2, *}$ & $0.056^{2}$ \\
\hline Flynn score (continuous) & $<0.001^{3, *}$ & $<0.001^{3, *}$ & $<0.001^{3, *}$ & $<0.001^{1, *}$ & $0.207^{1}$ \\
\hline $\begin{array}{l}\text { Admission C-reactive protein level (mg/dL) } \\
\text { (continuous) }\end{array}$ & $0.001^{3, *}$ & $<0.001^{3, *}$ & $<0.001^{3, *}$ & $<0.001^{1, *}$ & $0.155^{1}$ \\
\hline $\begin{array}{l}\text { Admission white blood cell count }(\times 1,000 \\
\text { cells } / \mu \mathrm{L}) \text { (continuous) }\end{array}$ & $<0.001^{3, *}$ & $<0.001^{3, *}$ & $<0.001^{3, *}$ & $<0.001^{1, *}$ & $0.018^{1, *}$ \\
\hline $\begin{array}{l}\text { Admission blood glucose level (mg/dL) } \\
\text { (continuous) }\end{array}$ & $0.001^{3, *}$ & $0.001^{3, *}$ & $0.695^{3}$ & $0.266^{1}$ & $0.808^{1}$ \\
\hline Admission temperature (binary) & $0.126^{1}$ & $0.009^{1, *}$ & $0.036^{1, *}$ & $0.143^{2}$ & $0.699^{2}$ \\
\hline Peak temperature during stay (binary) & $<0.001^{1, *}$ & $<0.001^{1, *}$ & $<0.001^{1, *}$ & $<0.001^{2}, *$ & $0.066^{2}$ \\
\hline Antibiotic regimen (categorical) & $<0.001^{7}, *$ & $<0.001^{7}, *$ & $<0.001^{7}, *$ & $<0.001^{6 * *}$ & $0.002^{6, *}$ \\
\hline I\&D (binary) & $<0.001^{1, *}$ & $<0.001^{1, *}$ & $0.026^{1, *}$ & $0.003^{2 *} *$ & $0.452^{2}$ \\
\hline Tracheostomy (binary) & $0.001^{1, *}$ & $<0.001^{1, *}$ & $0.001^{1, *}$ & $<0.001^{2} * *$ & $>0.999^{2}$ \\
\hline Surgery after admission (binary) & $<0.001^{1, *}$ & $<0.001^{1, *}$ & $<0.001^{1, *}$ & $<0.001^{2, *}$ & $0.758^{2}$ \\
\hline GA (binary) & $<0.001^{1, *}$ & $<0.001^{1, *}$ & $<0.001^{1, *}$ & $<0.001^{2, *}$ & $0.383^{2}$ \\
\hline Additional surgery (binary) & $<0.001^{1, *}$ & $<0.001^{1, *}$ & $0.129^{1}$ & $0.005^{2} *$ & $0.440^{2}$ \\
\hline Additional trip to the OR (binary) & $<0.001^{4, *}$ & $<0.001^{4, *}$ & $<0.001^{4, *}$ & $<0.001^{2, *}$ & $0.091^{2}$ \\
\hline No. of OR visits (continuous) & $<0.001^{3, *}$ & $<0.001^{3, *}$ & $<0.001^{3, *}$ & $<0.001^{1, *}$ & $0.158^{1}$ \\
\hline Length of operation under GA (continuous) & $<0.001^{3}, *$ & $<0.001^{3, *}$ & $0.008^{3, *}$ & $<0.001^{1, *}$ & $0.779^{1}$ \\
\hline No. of operations performed (continuous) & $<0.001^{3, *}$ & $<0.001^{3, *}$ & $0.034^{3, *}$ & $0.008^{1, *}$ & $0.830^{1}$ \\
\hline Operation type (categorical) & $<0.001^{7}, *$ & $<0.001^{7, *}$ & $0.001^{7}, *$ & $<0.001^{6 * *}$ & $0.611^{6}$ \\
\hline Location of surgery (categorical) & $<0.001^{7}$ & $<0.001^{7}$ & $<0.001^{7}$ & $<0.001^{6 * *}$ & $0.234^{6}$ \\
\hline ICU use (binary) & $<0.001^{1, *}$ & $<0.001^{1, *}$ & $<0.001^{1, *}$ & & $<0.001^{2, *}$ \\
\hline Length of stay in ICU (continuous) & $<0.001^{3, *}$ & $<0.001^{3, *}$ & $<0.001^{3, *}$ & & $0.070^{1}$ \\
\hline
\end{tabular}

(ICU: intensive care unit, DM: diabetes mellitus, I\&D: incision and drainage, GA: general anesthesia, OR: operation room)

$* P<0.05$.

$P$-value calculated by ${ }^{1}$ simple $t$-test, ${ }^{2}$ Pearson's chi-square test, ${ }^{3}$ Pearson correlation analysis, ${ }^{4}$ Mann-Whitney test, ${ }^{5}$ Kruskal-Wallis test (post-hoc analysis with Mann-Whitney), ${ }^{6}$ Pearson's chi-square test for each categorical group versus other subjects, ${ }^{7}$ one-way ANOVA (post-hoc analysis with Tukey test).

Hye-Won Kim et al: Factors associated with treatment outcomes of patients hospitalized with severe maxillofacial infections at a tertiary center. J Korean Assoc Oral Maxillofac Surg 2021

Significant variables in the initial univariable analysis were entered into the preliminary multiple regression model. To adjust for clustering of outcomes, the number of infected spaces, medical compromise, diabetes, incision and drainage, GA, additional surgery, and ICU use were eliminated from the multiple regression models for LOS, total hospital bill, average bill per one admission day, and ICU use.

Results of the final multivariate regression models after removal of variables that were no longer significant after controlling for other variables are summarized in Tables 3-7. Multivariable regression analysis revealed that $85.8 \%$ of the variation in length of hospital stay was due to different admission years, infection location, Flynn score, presence of deep neck infection, cardiovascular disease, admission CRP level, serum glucose level, length and number of surgeries, tracheostomy, time from admission to first surgery, and length of ICU stay.(Table 3) The variables of age, type of insurance, admission year, number of comorbidities, Flynn score, ad- 
Table 3. Final multiple linear regression analysis for length of stay

\begin{tabular}{lccc}
\hline \multicolumn{1}{c}{ Value name } & $\beta$ coefficient & $95 \%$ CI & $P$-value \\
\hline Constant) & -5.527 & -9.016 to -2.038 & $0.002^{*}$ \\
Admission year & Ref. & N/A & N/A \\
2011-2012 & 3.591 & 1.087 to 4.337 & $<.001^{*}$ \\
2017-2018 & 2.712 & 1.960 to 5.222 & $0.001^{*}$ \\
2015-2016 & & N/A & \\
Infection location & Ref. & 0.157 to 5.142 & $0.037^{*}$ \\
Both & 2.65 & $0.001^{*}$ \\
Single & 0.625 & -6.037 to 0.912 & $0.001^{*}$ \\
Flynn score & -3.765 & 2.010 to 4.624 & $<0.001^{*}$ \\
Presence of deep neck infection & 3.317 & $0.001^{*}$ \\
Presence of cardiovascular disease & 0.139 & 0.065 to 0.213 & $0.005^{*}$ \\
Admission C-reactive protein level & 0.013 & 0.004 to 0.022 & $<0.001^{*}$ \\
Admission blood glucose level & 0.064 & 0.042 to 0.086 & $0.001^{*}$ \\
Length of operation under GA & 2.647 & 1.311 to 3.983 & $0.002^{*}$ \\
No. of operations performed & 6.952 & 2.616 to 11.287 & $<0.001^{*}$ \\
Tracheostomy performed & 0.99 & 0.620 to 1.360 & $<0.001^{*}$ \\
Time from admission to first surgery & 0.66 & 0.534 to 0.786 & \\
Length of ICU stay & 0.858 & & \\
$R$ value & &
\end{tabular}

(CI: confidence interval, Ref.: reference, N/A: not applicable, GA: general anesthesia, ICU: intensive care unit)

$* P<0.05$.

The following variables were eliminated from the multivariable analysis model to adjust for clustering of outcomes: number of infected spaces, medically compromised, diabetes mellitus, incision and drainage, GA performed, additional surgery performed, ICU use.

Hye-Won Kim et al: Factors associated with treatment outcomes of patients hospitalized with severe maxillofacial infections at a tertiary center. J Korean Assoc Oral Maxillofac Surg 2021

Table 4. Final multiple linear regression analysis for total amount billed ( $\log _{2}$ value)

\begin{tabular}{|c|c|c|c|}
\hline Value name & $\beta$ coefficient & $95 \% \mathrm{CI}$ & $P$-value \\
\hline (Constant) & 20.091 & 19.420 to 20.761 & $<0.001 *$ \\
\hline Age & 0.008 & 0.005 to 0.012 & $<0.001 *$ \\
\hline \multicolumn{4}{|l|}{ Type of insurance } \\
\hline Not insured & Ref. & N/A & N/A \\
\hline Medial aid & -1.173 & -1.833 to -0.513 & $0.001 *$ \\
\hline NHI & -0.793 & -1.407 to -0.179 & $0.012 *$ \\
\hline \multicolumn{4}{|l|}{ Admission year } \\
\hline 2011-2012 & Ref. & N/A & N/A \\
\hline $2019-2020$ & 0.578 & 0.413 to 0.744 & $<0.001 *$ \\
\hline $2017-2018$ & 0.524 & 0.359 to 0.689 & $<0.001 *$ \\
\hline $2015-2016$ & 0.292 & 0.124 to 0.459 & $0.001 *$ \\
\hline No. of comorbidities & 0.082 & 0.027 to 0.136 & $0.003 *$ \\
\hline Flynn score & 0.037 & 0.015 to 0.060 & $0.001 *$ \\
\hline Admission C-reactive protein level & 0.02 & 0.013 to 0.026 & $<0.001^{*}$ \\
\hline Admission white blood cell count & 0.019 & 0.007 to 0.031 & $0.002 *$ \\
\hline Admission blood glucose level & 0.001 & 0.000 to 0.002 & $0.038 *$ \\
\hline Admission body temperature $>37.5^{\circ} \mathrm{C}$ & -0.201 & -0.388 to -0.014 & $0.035 *$ \\
\hline Peak body temperature $>37.5^{\circ} \mathrm{C}$ & 0.321 & 0.163 to 0.479 & $<0.001 *$ \\
\hline Surgery performed after admission & 0.76 & 0.552 to 0.968 & $<0.001 *$ \\
\hline \multicolumn{4}{|l|}{ Type of surgery } \\
\hline I\&D alone & Ref. & N/A & N/A \\
\hline Other surgery without I\&D & -0.42 & -0.828 to -0.013 & $0.043 *$ \\
\hline Length of surgery under GA & 0.005 & 0.003 to 0.007 & $<0.001 *$ \\
\hline \multicolumn{4}{|l|}{ I\&D location } \\
\hline Operation room & Ref. & N/A & N/A \\
\hline Outpatient clinic after admission & -0.569 & -0.764 to -0.374 & $<0.001^{*}$ \\
\hline ER & -0.465 & -0.703 to -0.227 & $<0.001 *$ \\
\hline Tracheostomy performed & 0.595 & 0.218 to 0.972 & $0.002 *$ \\
\hline Time from admission to first surgery & 0.069 & 0.032 to 0.106 & $<0.001^{*}$ \\
\hline Length of ICU stay & 0.031 & 0.018 to 0.043 & $<0.001 *$ \\
\hline$R$ value & 0.904 & & \\
\hline
\end{tabular}

(CI: confidence interval, Ref.: reference, N/A: not applicable, NHI: National Health Insurance, I\&D: incision and drainage, GA: general anesthesia, ER: emergency room, ICU: intensive care unit)

$* P<0.05$.

The following variables were eliminated from the multivariable analysis model to adjust for clustering of outcomes: number of infected spaces, medically compromised, diabetes mellitus, incision and drainage, GA performed, additional surgery performed, ICU use.

Hye-Won Kim et al: Factors associated with treatment outcomes of patients hospitalized with severe maxillofacial infections at a tertiary center. J Korean Assoc Oral Maxillofac Surg 2021

mission CRP, WBC, serum glucose, admission temperature, peak temperature, surgery after admission, type, length, and location of surgery, tracheostomy, time from admission to first surgery, and length of ICU stay accounted for $90.4 \%$ of the variation in the total hospital bill.(Table 4) Significant variables accounting for $73.8 \%$ of the variation in the average 
Table 5. Final multiple linear regression analysis for average bill per one admission day ( $\log _{2}$ value)

\begin{tabular}{|c|c|c|c|}
\hline Value name & $\beta$ coefficient & $95 \% \mathrm{CI}$ & $P$-value \\
\hline (Constant) & 18.67 & 18.237 to 19.102 & $<0.001^{*}$ \\
\hline Age & 0.002 & 0.000 to 0.004 & $0.019 *$ \\
\hline \multicolumn{4}{|l|}{ Type of insurance } \\
\hline Not insured & Ref. & N/A & N/A \\
\hline Medical aid & -1.198 & -1.618 to -0.779 & $<0.001 *$ \\
\hline NHI & -0.991 & -1.379 to -0.603 & $<0.001 *$ \\
\hline \multicolumn{4}{|l|}{ Admission year } \\
\hline $2011-2012$ & Ref. & N/A & N/A \\
\hline 2013-2014 & 0.153 & 0.018 to 0.289 & $0.027 *$ \\
\hline $2015-2016$ & 0.212 & 0.081 to 0.343 & $0.002 *$ \\
\hline $2017-2018$ & 0.277 & 0.146 to 0.408 & $<0.001 *$ \\
\hline $2019-2020$ & 0.825 & 0.699 to 0.951 & $<0.001 *$ \\
\hline Presence of deep neck infection & 0.174 & 0.052 to 0.297 & $0.005^{*}$ \\
\hline Admission white blood cell count & 0.012 & 0.004 to 0.020 & $0.004 *$ \\
\hline Peak body temperature $>37.5^{\circ} \mathrm{C}$ & 0.117 & 0.026 to 0.208 & $0.012 *$ \\
\hline No. of operations under GA & 0.184 & 0.094 to 0.275 & $<0.001 *$ \\
\hline \multicolumn{4}{|l|}{ Location of surgery } \\
\hline OR & Ref. & N/A & N/A \\
\hline Outpatient clinic before admission & -0.148 & -0.261 to -0.035 & $0.011 *$ \\
\hline $\mathrm{ER}$ & 0.181 & 0.037 to 0.325 & $0.014 *$ \\
\hline Length of ICU stay & 0.016 & 0.009 to 0.023 & $<0.001 *$ \\
\hline$R$ value & 0.738 & & \\
\hline
\end{tabular}

(CI: confidence interval, Ref.: reference, N/A: not applicable, NHI: National Health Insurance, GA: general anesthesia, OR: operating room, ER: emergency room, ICU: intensive care unit)

$* P<0.05$.

The following variables were eliminated from the multivariable analysis model to adjust for clustering of outcomes: number of infected spaces, medically compromised, incision and drainage, GA performed, ICU use.

Hye-Won Kim et al: Factors associated with treatment outcomes of patients hospitalized with severe maxillofacial infections at a tertiary center. J Korean Assoc Oral Maxillofac Surg 2021

Table 6. Final multiple logistic regression analysis for use of ICU

\begin{tabular}{lccc}
\hline \multicolumn{1}{c}{ Value name } & $\beta$ coefficient & $95 \%$ CI & $P$-value \\
\hline (Constant) & -5.947 & N/A & $<0.001^{*}$ \\
Age & 1.029 & 0.995 to 1.063 & 0.094 \\
Route of admission & $<0.001$ & $<0.001$ & 0.996 \\
ER & Ref. & N/A & N/A \\
Side & 0.157 & 0.036 to 0.687 & $0.014^{*}$ \\
Both & 1.787 & $<.004^{*}$ \\
Single & 10.136 & 1.202 to 2.655 & $<0.001^{*}$ \\
No. of comorbidities & 1.047 & 2.922 to 35.164 & $<0.001^{*}$ \\
Peak body temperature $>37.5^{\circ} \mathrm{C}$ & & 1.027 to 1.067 & N/A \\
Length of operation under GA & Nef. & $0.025^{*}$ \\
Type of surgery & 0.015 & $<0.001$ to 0.591 & 0.997 \\
I\&D alone & 2.61 & $<0.001$ & \\
Tracheostomy performer & 0.684 & & \\
Nagelkerke $R^{2}$ value & & \\
\hline
\end{tabular}

(ICU: intensive care unit, CI: confidence interval, ER: emergency room, Ref.: reference, N/A: not applicable, GA: general anesthesia, I\&D: incision and drainage)

$* P<0.05$.

The following variables were eliminated from the multivariable analysis model to adjust for clustering of outcomes: number of infected spaces, medically compromised, I\&D, GA performed, additional surgery performed.

Hye-Won Kim et al: Factors associated with treatment outcomes of patients hospitalized with severe maxillofacial infections at a tertiary center. J Korean Assoc Oral Maxillofac Surg 2021

Table 7. Final multiple logistic regression analysis for other outcomes versus complete recovery

\begin{tabular}{lccc}
\hline \multicolumn{1}{c}{ Value name } & $\beta$ coefficient & 95\% CI & $P$-value \\
\hline Constant) & -8.16 & N/A & $<0.001^{*}$ \\
Age & 1.064 & 1.012 to 1.118 & $0.014^{*}$ \\
Use of ICU & 7.727 & 2.239 to 26.666 & $0.001^{*}$ \\
Nagelkerke $R^{2}$ value & 0.253 & & \\
\hline
\end{tabular}

(CI: confidence interval, N/A: not applicable, ICU: intensive care unit) $* P<0.05$.

Hye-Won Kim et al: Factors associated with treatment outcomes of patients hospitalized with severe maxillofacial infections at a tertiary center. J Korean Assoc Oral Maxillofac Surg 2021 bill per one admission day were age, insurance, admission year, deep neck infection, admission WBC, peak temperature, number and location of surgeries performed, and length of ICU stay.(Table 5) Only infection location, number of comorbidities, peak temperature, and length and type of surgery had a significant association with stay in the ICU in the final multiple logistic regression analysis. Age, route of admission, and performance of tracheostomy were included in the final regression model, but had $P$-values higher than 0.05 and were 
thus considered to be non-significant variables.(Table 6) The only two variables that showed a significant association with discharge outcomes in the final multiple logistic regression analysis were age and ICU use.(Table 7)

\section{Discussion}

In this retrospective study, we sought to describe the demographics, treatment outcomes, and hospital costs of patients with severe maxillofacial infections requiring admission at a tertiary center in South Korea and to assess the associations between treatment outcomes and diverse social, patient health, and treatment-related variables. To the best of our knowledge, this is the first study to document the financial outcomes and associated factors of patients hospitalized for maxillofacial infections in an Asian country.

\section{Demographic characteristics}

Mean age of occurrence for severe maxillofacial infections requiring hospitalization was 54.69 years. This is a significantly older age than reported in previous studies ${ }^{1-3,5-8,11-18}$, which reported severe maxillofacial infections around the fourth and fifth decades of life. This is also higher than the reported mean age of 47.13 years in a previous Korean study by Park and colleagues on maxillofacial patients who presented to the $\mathrm{ER}^{17}$. The relatively older average age of the patients in the present study can be attributed to the rapid aging of the Korean population in the recent years, which has been reported to be the fastest among OECD (Organisation for Economic Co-operation and Development) countries, with the share of the population aged 65 and above expected to exceed one-third by $2050^{19}$. Therefore, it is essential that surgeons pay special attention to the oral health care of the older members of society as they are at higher risk of developing severe maxillofacial infections.

Many patients had multiple comorbidities. The high rate of patients with at least one comorbid condition (59\%) is in accordance with the findings of previous studies ${ }^{1,2,5}$. In light of these findings, Abramowicz et al. ${ }^{1}$ recommended that orofacial infections in medically compromised patients be identified as early as possible to make the early delivery of antibiotics and surgical intervention possible.

Most cases in the current study (97.2\%) were due to odontogenic causes. This is in accordance with a previous Korean review that reported the rate of maxillofacial infections due to odontogenic infections in Korea to be $92.47 \%{ }^{17}$. However, this figure is much higher than those previously documented by other researchers for hospitalized patients of $61.8 \%$ to $71.0 \%{ }^{7,13,14,16,20}$. This is probably due to the nature of the present study, in which only patients admitted to the oral and maxillofacial department were included in the statistical analysis. To focus on non-odontogenic infections, future studies should also include patients admitted to the ENT department.

\section{Treatment outcomes}

Average LOS in this study was 11.47 days. While this is in accordance with studies conducted in South Korea ${ }^{17,21,22}$ and other Eastern countries ${ }^{18,20}$, this was a nearly two- to four-fold higher rate than the figures reported for Western populations. A previous study based on national data that investigated the treatment outcomes of patients in the USA admitted with mouth cellulitis reported an average LOS of only 3.9 days ${ }^{12}$. Other Western studies reported similar results, with the mean LOS ranging from 2.96 to 5.46 days s, $2,5,9,10,23^{\text {. }}$

However, despite the overwhelmingly longer period of hospital stay observed among our population, the mean hospital expenditure was only $\$ 4,216.67$. This is in stark contrast to the reported mean hospital charges in previous USA reports of $\$ 14,245$ to $\$ 48,351^{1,2,5,6,8-10,12,23}$. This figure is also lower than that reported in one previous British study on the cost outcomes of hospitalization of deep neck infections, which is the only study in the literature that documents the hospital costs related to maxillofacial infections outside of the USA ${ }^{7}$. The authors of that study reported a mean LOS of 11 days and a mean hospital charge of $\$ 7,562^{7}$.

The prolonged admission period and low treatment costs of the current study can be attributed to Korea's unique NHI Program and the accessibility of medical services. The NHI Program is a universal social insurance program that oversees all health care in the Republic of Korea. It is compulsory by law, and thus covers the entire population residing in the Korean domain ${ }^{24,25}$. It is operated by a single insurer, the NHI Service (NHIS), which operates under supervision of the Korean government. Under this insurance program, the entire population is entitled to only a portion of medical utilization $\operatorname{costs}^{24}$. Approximately $3.7 \%$ of the population in the low-income bracket are also assisted by a free medical aid program and can use medical services free of charge ${ }^{25}$. South Korea thus boasts $100 \%$ accessibility to necessary health care services, which is in stark contrast to the accessibility value reported for the United States of $90.8 \%$ as reported by the OECD in $2019^{19}$. The high ratio (99\%) of the insured popula- 
tion in the current study implies exceptional access to medical services, in contrast to the values reported in previous studies in the USA of $16.9 \%$ to $82.5 \%^{1,2,5,10,12,23}$.

The substantially lower costs reported in this study are also consistent with medical costs in South Korea reported in previous OECD reports, in which the comparative average price level for health was only $48 \%$ of that of the USA and $24 \%$ lower than the OECD average health $\operatorname{cost}^{19}$.

However, other recent OECD reviews of the South Korean medical system have all identified over-provision of hospital admissions to be a major quality issue ${ }^{26}$. Sohn and Jung ${ }^{24}$ attributed this phenomenon to the 'moral hazard' model, which refers to the trend in which patients use medical services more than is necessary or are negligent in health promotion due to low out-of-pocket costs. The significantly longer duration of hospital stay in the current study compared to previous studies may also be due to this trend. Therefore, future treatment of maxillofacial infections in the South Korean population should focus on the prevention of severe infections and the establishment of efficient treatment protocols such as prompt surgical interventions.

In our study, the vast majority of cases were routinely discharged following complete recovery (96.9\%), and the mortality rate was only $1.0 \%$. This recovery rate is substantially higher than the $88 \%$ documented in a 2008 USA study based on nationwide data ${ }^{12}$. This relatively high rate of recovery may also be due to the ease of access and relatively low burden of Korean medical services. Because most patients are covered by the NHI, patients may present to the hospital earlier in their disease course, a more aggressive treatment approach may be followed, and length of care is not a consideration, all of which would increase the probability of complete resolution of the infection. A similar finding was reported in a previous study by Yew et al. ${ }^{16}$, who attributed the low rates of severe complications and mortality among patients with orofacial infections in their study to the affordability and accessibility of the Malaysian public healthcare service. The poor oral health status and treatment outcomes among populations at the lower end of the socioeconomic scale, and the need for improved access to healthcare for this socioeconomic group have been emphasized in a recent report by Kruger and Tennant $^{27}$.

\section{Factors significantly associated with treatment outcomes}

Although many researchers have sought to identify variables associated with the treatment outcomes of maxillofacial infections via univariable analysis, few studies have used multivariable analysis to identify potential relevant prognostic factors. We argue that the outcomes of maxillofacial infections cannot be predicted by one factor alone but are likely affected by patient-, disease-, and treatment-related factors. The complex nature of maxillofacial infections is highlighted in this study by our finding that most independent variables were significantly associated with outcome variables in initial univariable analysis but were later excluded after controlling for other variables.

In this study, $85.8 \%$ of the total variation in LOS was explained by the multivariable regression model, which is a relatively higher prediction value than those reported previously of $32.8 \%$ to $68.2 \% \%^{3,13}$. The rates of variation in total hospital bill (90.4\%), average hospital bill per day (73.8\%), and prevalence of ICU stay $(68.4 \%)$ in the final regression analysis models were also relatively higher than reported in similar studies ${ }^{3,23}$. The multivariable model in the study by Flynn et al. ${ }^{28}$ reported a similar ratio of variability in LOS (84\%) to that reported in this study, but the study population comprised only 37 subjects.

Not surprisingly, variables related to involvement of multiple anatomical sites such as infection side, Flynn score, and presence of deep neck infection were significantly correlated with longer LOS and higher costs. The association between multi-space infections and longer stay in the hospital in addition to higher hospital bills has been documented in several previous reports ${ }^{3,12,17,28}$. We also found that a delay in surgical intervention was associated with a longer stay, indicating the importance of prompt surgery when deemed necessary.

Of note is our finding that admission serum glucose level was significantly associated with overall LOS and hospital costs. The significant correlation between elevated serum glucose level due to diabetes and longer hospital stay, increased possibility of severe complications, and poorer treatment outcomes of maxillofacial infection patients has been documented in several previous studies ${ }^{3,12,17,20}$. This relationship has been attributed to poor tissue perfusion with a subsequent delay in wound healing and immune system dysregulation ${ }^{5,20}$. Other health-related variables such as elevated WBC level and body temperature were also associated with increased hospital costs. 
Factors that were significantly associated with ICU stay were the location of infection, number of comorbidities, peak temperature, length of operation, and type of surgery. Similar correlations were reported by Christensen et al. ${ }^{3}$ who also performed a multivariate logistic regression analysis.

In the present study, $25.3 \%$ of discharge outcomes other than complete recovery could be predicted by the final multiple logistic regression analysis model. Although nearly $75 \%$ of unfavorable discharge outcomes could not be explained, age and use of the ICU were significantly associated with an unfavorable discharge outcome. Among these two variables, stay in the ICU is an unavoidable complication of a severe infection. Our study results therefore suggest that older age is the only significant variable associated with a poor treatment outcome. Meticulous attention must thus be paid when treating the older population suffering from severe maxillofacial infections as this population is at increased risk of life-threatening complications, prolonged stay in the ICU, and death.

\section{Limitations and strengths}

As a retrospective chart review, this study has several limitations. Due to its retrospective nature, the possibility of misleading or inaccurate records cannot be ruled out. Furthermore, as mentioned above, we were only able to demonstrate significant associations between variables and outcomes, not cause and effect. Furthermore, the study was limited to patients treated in a single tertiary center and may therefore not be nationally representative.

Despite these shortcomings, the current study is the first study to investigate factors associated with the hospital costs of maxillofacial infections in an Asian country based on multivariable analysis of a relatively heterogeneous population of 390 patients.

\section{Conclusion}

Although the mean total hospital expenditure for patients with maxillofacial infections was much lower in our South Korean patient population than reported for Western countries, the mean length of hospital stay was much longer, and the rate of complete recovery much higher, suggesting overprovision of hospital services for the treatment of maxillofacial infections. This emphasizes the importance of optimizing accessibility to medical services to obtain the best treatment outcomes.

For patients hospitalized with maxillofacial infections, the following should be taken into consideration.

1. To limit LOS, meticulous attention must be paid to the timing, duration, and number of appropriate surgical interventions, appropriate ICU use, and overall determination of the patient's health status and infection severity.

2. Hospital costs are significantly influenced by factors related to insurance, patient's initial status upon admission, ICU use, and the timing, duration, type, location, and number of appropriate surgical interventions.

3. Special attention must be paid when treating old patients suffering from maxillofacial infections, for the only key variable impacting treatment outcome is patient age.

\section{ORCID}

Hye-Won Kim, https://orcid.org/0000-0002-5959-4080

Chul-Hwan Kim, https://orcid.org/0000-0002-5199-2420

\section{Authors' Contributions}

H.W.K. participated in data collection, performed the statistical analysis, and wrote the manuscript. C.H.K. participated in the study design and coordination and helped to draft the manuscript. All authors read and approved the final manuscript.

\section{Ethics Approval and Consent to Participate}

The study was approved by the Institutional Review Board of Dankook Dental University Hospital (No. DKUDH IRB 2020-09-007), and the informed consent was waived by the IRB.

\section{Conflict of Interest}

No potential conflict of interest relevant to this article was reported.

\section{References}

1. Abramowicz S, Rampa S, Allareddy V, Lee MK. The burden of facial cellulitis leading to inpatient hospitalization. J Oral Maxillofac Surg 2017;75:1656-67. https://doi.org/10.1016/j.joms.2017.01.032

2. Eisler L, Wearda K, Romatoski K, Odland RM. Morbidity and cost of odontogenic infections. Otolaryngol Head Neck Surg 2013;149:84-8. https://doi.org/10.1177/0194599813485210

3. Christensen B, Han M, Dillon JK. The cause of cost in the management of odontogenic infections 2: multivariate outcome analyses. J Oral Maxillofac Surg 2013;71:2068-76. https://doi.org/10.1016/ j.joms.2013.05.027 
4. Flynn TR, Shanti RM, Levi MH, Adamo AK, Kraut RA, Trieger N. Severe odontogenic infections, part 1: prospective report. J Oral Maxillofac Surg 2006;64:1093-103. https://doi.org/10.1016/ j.joms.2006.03.015

5. Gams K, Shewale J, Demian N, Khalil K, Banki F. Characteristics, length of stay, and hospital bills associated with severe odontogenic infections in Houston, TX. J Am Dent Assoc 2017;148:2219. https://doi.org/10.1016/j.adaj.2016.11.033

6. Ahmad N, Abubaker AO, Laskin DM, Steffen D. The financial burden of hospitalization associated with odontogenic infections. J Oral Maxillofac Surg 2013;71:656-8. https://doi.org/10.1016/ j.joms.2012.11.024

7. Hurley RH, Douglas CM, Montgomery J, Clark LJ. The hidden cost of deep neck space infections. Ann R Coll Surg Engl 2018;100:129-34. https://doi.org/10.1308/rcsann.2017.0193

8. Allareddy V, Rampa S, Nalliah RP, Allareddy V. Longitudinal discharge trends and outcomes after hospitalization for mouth cellulitis and Ludwig angina. Oral Surg Oral Med Oral Pathol Oral Radiol 2014;118:524-31. https://doi.org/10.1016/j.oooo.2014.06.003

9. Jundt JS, Gutta R. Characteristics and cost impact of severe odontogenic infections. Oral Surg Oral Med Oral Pathol Oral Radiol 2012;114:558-66. https://doi.org/10.1016/j.oooo.2011.10.044

10. Shah AC, Leong KK, Lee MK, Allareddy V. Outcomes of hospitalizations attributed to periapical abscess from 2000 to 2008: a longitudinal trend analysis. J Endod 2013;39:1104-10. https://doi. org/10.1016/j.joen.2013.04.042

11. Kim MK, Allareddy V, Nalliah RP, Kim JE, Allareddy V. Burden of facial cellulitis: estimates from the Nationwide Emergency Department Sample. Oral Surg Oral Med Oral Pathol Oral Radiol 2012;114:312-7. https://doi.org/10.1016/j.tripleo.2011.07.043

12. Kim MK, Nalliah RP, Lee MK, Allareddy V. Factors associated with length of stay and hospital charges for patients hospitalized with mouth cellulitis. Oral Surg Oral Med Oral Pathol Oral Radiol 2012;113:21-8. https://doi.org/10.1016/j.tripleo.2011.01.012

13. Jansisyanont P, Kasemsai W, Bamroong P. Factors related to the treatment outcome of maxillofacial fascia space infection. $\mathrm{J}$ Oral Maxillofac Surg Med Pathol 2015;27:458-64. https://doi. org/10.1016/j.ajoms.2014.04.009

14. Zhang C, Tang Y, Zheng M, Yang J, Zhu G, Zhou H, et al. Maxillofacial space infection experience in West China: a retrospective study of 212 cases. Int J Infect Dis 2010;14:e414-7. https://doi. org/10.1016/j.ijid.2009.08.002

15. Mathew GC, Ranganathan LK, Gandhi S, Jacob ME, Singh I, Solanki M, et al. Odontogenic maxillofacial space infections at a tertiary care center in North India: a five-year retrospective study. Int J Infect Dis 2012;16:e296-302. https://doi.org/10.1016/ j.ijid.2011.12.014

16. Yew CC, Ng MP, Ling XF, Tew MM. Orofacial infection and influencing factors on prolonged hospital stay: a four year retrospective study of 207 cases. J Oral Maxillofac Surg Med Pathol 2021;33:7-
12. https://doi.org/10.1016/j.ajoms.2020.06.007

17. Park J, Lee JY, Hwang DS, Kim YD, Shin SH, Kim UK, et al. A retrospective analysis of risk factors of oromaxillofacial infection in patients presenting to a hospital emergency ward. Maxillofac Plast Reconstr Surg 2019;41:49. https://doi.org/10.1186/s40902019-0238-9

18. Agarwal AK, Sethi A, Sethi D, Mrig S, Chopra S. Role of socioeconomic factors in deep neck abscess: a prospective study of 120 patients. Br J Oral Maxillofac Surg 2007;45:553-5. https://doi. org/10.1016/j.bjoms.2007.01.001

19. OECD. Health at a glance 2019: OECD indicators. Paris: OECD Publishing; 2019.

20. Zheng L, Yang C, Zhang W, Cai X, Kim E, Jiang B, et al. Is there association between severe multispace infections of the oral maxillofacial region and diabetes mellitus? J Oral Maxillofac Surg 2012;70:1565-72. https://doi.org/10.1016/j.joms.2011.07.010

21. Jang JW, Kim CH, Kim MY. Analysis of glycosylated hemoglobin (HbA1c) level on maxillofacial fascial space infection in diabetic patients. J Korean Assoc Oral Maxillofac Surg 2015;41:251-8. https://doi.org/10.5125/jkaoms.2015.41.5.251

22. Kang SK, Lee S, Oh HK, Kang MW, Na MH, Yu JH, et al. Clinical features of deep neck infections and predisposing factors for mediastinal extension. Korean J Thorac Cardiovasc Surg 2012;45:1716. https://doi.org/10.5090/kjtcs.2012.45.3.171

23. Christensen B, Han M, Dillon JK. The cause of cost in the management of odontogenic infections 1: a demographic survey and multivariate analysis. J Oral Maxillofac Surg 2013;71:2058-67. https:// doi.org/10.1016/j.joms.2013.05.026

24. Sohn M, Jung M. Effects of public and private health insurance on medical service utilization in the National Health Insurance System: national panel study in the Republic of Korea. BMC Health Serv Res 2016;16:503. https://doi.org/10.1186/s12913-016-1746-2

25. Song YJ. The South Korean health care system. JMAJ 2009;52: 206-9.

26. OECD. OECD Reviews of public health. Paris: OECD; 2020.

27. Kruger E, Tennant M. Socioeconomic disadvantage and oralhealth-related hospital admissions: a 10-year analysis. BDJ Open 2016;2:16004. https://doi.org/10.1038/bdjopen.2016.4

28. Flynn TR, Shanti RM, Hayes C. Severe odontogenic infections, part 2: prospective outcomes study. J Oral Maxillofac Surg 2006;64:1104-13. https://doi.org/10.1016/j.joms.2006.03.031

How to cite this article: Kim HW, Kim CH. Factors associated with treatment outcomes of patients hospitalized with severe maxillofacial infections at a tertiary center. J Korean Assoc Oral Maxillofac Surg 2021;47:197-208. https://doi.org/10.5125/jkaoms.2021.47.3.197 